Research Paper

\title{
Palliative beam radiotherapy offered real-world survival benefit to metastatic rectal cancer: A large US population-based and propensity score-matched study
}

\author{
Qi Liu ${ }^{1,2^{*}}$, Zezhi Shan ${ }^{1,2^{*}}$, Dakui Luo ${ }^{1,2}$, Sanjun Cai1,2, Qingguo $\mathrm{Li}^{1,2}{ }^{\circledR}$, and Xinxiang $\mathrm{Li}^{1,2^{\circledR}}$ \\ 1. Department of Colorectal Surgery, Fudan University Shanghai Cancer Center, Shanghai, China \\ 2. Department of Oncology, Shanghai Medical College, Fudan University, Shanghai, China. \\ ${ }^{*}$ Qi Liu and Zezhi Shan contributed equally to this work. \\ $\triangle$ Corresponding authors: Xinxiang Li MD, PhD, Department of Colorectal Surgery, Fudan University Shanghai Cancer Center, \#270 Dongan Road, Xuhui \\ District, Shanghai, China, Postal code: 200032, E-mail: 11491xx@sina.com; Qingguo Li MD, PhD, Department of Colorectal Surgery, Fudan University Shanghai \\ Cancer Center, \#270 Dongan Road, Xuhui District, Shanghai, China, Postal code: 200032, E-mail: oncosurgeonli@sohu.com \\ (c) Ivyspring International Publisher. This is an open access article distributed under the terms of the Creative Commons Attribution (CC BY-NC) license \\ (https://creativecommons.org/licenses/by-nc/4.0/). See http://ivyspring.com/terms for full terms and conditions.
}

Received: 2018.07.25; Accepted: 2018.12.23; Published: 2019.01.29

\begin{abstract}
Purpose: Radiotherapy (RT) has been reported to effectively palliate many symptoms of patients with metastatic rectal cancer ( $m R C)$. The objective of this study was to evaluate the survival benefit of $R T$ in $m R C$.

Methods: A retrospective population-based cohort study was performed using the Surveillance, Epidemiology, and End Results Program (SEER) database. Patient baseline demographic characteristics between the RT and no-RT groups were compared using Pearson chi-square tests. The outcome of interest was cause-specific survival (CSS). Propensity score (PS) matching and Cox proportional hazards regression analyses were performed to evaluate the prognostic power of variables on CSS.

Results: A total of 8851 patients with $\mathrm{mRC}$ were identified in the SEER database. Multivariable Cox regression analysis showed that $R T$ was a protective factor in $\mathrm{mRC}$ (hazard ratio $[H R]=0.702,95 \%$ confidence interval $[C l]=0.665-0.741, p<0.001)$. In subgroup analysis, multivariate Cox analysis demonstrated that patients of both surgery and no-surgery subgroups treated with RT had better CSS than those not treated with RT (HR $=0.654$, $95 \% \mathrm{Cl}=0.607-0.704, \quad \mathrm{p}<0.001$ for the surgery group; $\mathrm{HR}=0.779,95 \% \mathrm{Cl}=0.717-0.847, \mathrm{p}<0.001$ for the no-surgery group), PS matching resulted in $4170 \mathrm{mRC}$ patients and RT group presented significantly improved survival benefit than no-RT group ( 22.0 vs. $13.5 \%, \mathrm{P}<0.001)$. In surgery subgroup after PS matching, in especial, RT group showed more evidently improved survival benefit than no-RT group $(30.3$ vs. $18.0 \%, \mathrm{p}<0.001)$.

Conclusion: Using the SEER database, we definitely demonstrated that RT was associated with a significant survival advantage beyond the relief of a variety of pelvic symptoms in the setting of $\mathrm{mRC}$. This study strongly supports the use of RT in selected patients with $\mathrm{mRC}$, especially in patients who have undergone surgery. More studies need to be conducted to accurately define the role of RT in $\mathrm{mRC}$.
\end{abstract}

Key words: palliative; radiotherapy; metastatic rectal cancer; propensity score-matched

\section{Introduction}

Colorectal cancer (CRC) is the third most commonly diagnosed cancer in the United States [1]. The incidence of CRC in Asian countries is increasing rapidly, a trend that is similar to that seen in Western countries [2,3]. Approximately $20 \%-25 \%$ of patients will present with metastatic disease and an additional $20-30 \%$ of patients will develop metastatic disease after surgery [4, 5]. However, without treatment, these metastatic rectal cancer (mRC) patients will have a life expectancy of only between 5 months and 6 months in stark contrast to patients diagnosed without metastasis [6, 7]. Currently, the combination of surgical techniques, diagnostics, radiotherapy and new oncologic drugs has contributed to improved outcomes in rectal cancer (RC), including prolonged survival in mRC [8]. The overall survival of patients diagnosed with unresectable metastatic colorectal cancer has increased to more than 30 months with the 
integration of multiple cytotoxic agents and targeted therapies [9].

Although RT was included in the National Comprehensive Cancer Network (NCCN) guidelines as a treatment option for various types of cancer, population-based studies report a general underutilization of palliative radiotherapy [10]. However, the real-world effect of RT on survival of cancer patients might do exist and was worth exploring. In addition, metastatic nasopharyngeal cancer, metastatic prostate cancer and metastatic breast cancer are reported to have a survival benefit when treated with radiotherapy (RT) [11-13]. Therefore, we conducted this study using the Surveillance, Epidemiology, and End Results Program (SEER) database to determine whether mRC patients who received radiotherapy therapy had improved cause-specific survival (CSS) compared with those who did not.

\section{Materials and Methods}

\section{Patients}

Data were obtained for all patients with a diagnosis of malignant primary rectal cancer from the SEER Program of the National Cancer Institute (Figure 1). The SEER Program is an authoritative source of information on cancer incidence and survival in the United States. The SEER database provides a comprehensive source of population-based information and included all newly diagnosed cancer cases that occur in people residing in SEER-participating areas. Between 2004 and 2013, the SEER database included approximately $28 \%$ of the United States population. Patients were selected based on the following criteria: (1) patients were diagnosed from 2004 to 2013, (2) patients presented with $\mathrm{mRC}$, (3) patients had no history of other cancer, (4) patients were diagnosed with confirmed histology, (5) patients were under active follow-up, (6) TNM stage was known, (7) the surgery status of patients was known, and (8) information on CSS and survival months was available.

\section{Statistical analyses}

The different baseline characteristics between patients treated with RT and without RT were compared by Pearson chi-squared test for categorical variables. The endpoint used for comparison was rectal CSS based on selected patients. For univariate analysis, the Kaplan-Meier method with a log-rank test and a univariate Cox proportional hazards model were used. Age at diagnosis, gender, race, marital status at diagnosis, grade, T stage, $\mathrm{N}$ stage, histology, status of surgery, radiotherapy, and tumor size were included in the multivariate analysis by a Cox proportional hazards model.

In observational studies, there can be significant bias introduced by inherent differences between patients based on treatment selection. To decrease the risk of biased estimates of treatment effect, we defined the logit of predicted probability of treatment as a propensity score using the following baseline characteristics that strongly related to the survival but less strongly related to treatment: age at diagnosis, gender, race, marital status at diagnosis, grade, $\mathrm{T}$ stage, $\mathrm{N}$ stage, histology, status of surgery and tumor size $[14,15]$. Subjects receiving RT were matched on a one-to-one basis with subjects without receiving RT. Matching was performed based on nearest-neighbor matching, and RT and no-RT patients were matched within their respective risk groups. Propensity scores reflect the probability that patients received RT or did not receive RT based on their baseline characteristics.

\section{Results}

\section{Patient characteristics of overall cohort}

A total of 8851 patients with $\mathrm{mRC}$ were identified in the SEER database. Patients' baseline demographic characteristics are summarized in Table 1. In total, 5540 patients who did not receive RT and 3273 patients who did receive $\mathrm{RT}$ were included in the analyses. For the overall cohort, what is evident is that patients less than 65 years old were more likely to receive radiotherapy $(59.2 \%$ vs. $67.4 \%, \mathrm{p}<0.01)$. Besides, patients who had T3 stage disease $(53.9 \%$ vs. $58.6 \%, \mathrm{p}<0.01)$, had N1 stage disease ( $34.0 \%$ vs. $41.2 \%$, $\mathrm{p}<0.01)$, or had not undergone surgery $(35.7 \%$ vs. $40.9 \%, \mathrm{p}<0.01)$ were more likely to receive RT. Other characteristics were similar in the groups with and without RT. Propensity score (PS) matching was performed on the overall cohort, and resulted in 2085 patients in the no-RT group and 2085 patients in the RT group.

\section{Improved survival benefit of radiotherapy in mRC patients}

The median follow-up time for the overall cohort was 16 months. At the end of the follow-up time, $5916(66.8 \%)$ patients died of $\mathrm{mRC}$, and the 1-, 3-, and 5 -year CSS rates were $68.3 \%, 31.6 \%$, and $15.8 \%$, respectively.

The results of multivariate analyses by Cox regression is detailed in Table 2. RT was identified as a predictive factor for survival on univariate analysis (Figure 2A, HR=0.713, 95\% CI=0.675-0.752, $\mathrm{P}<0.001$ ). Factors that showed prognostic significance in univariate analysis were included in multivariate analysis of the overall cohort. When multivariate Cox regression analysis was performed, the outcome showed that RT was associated with a significant 


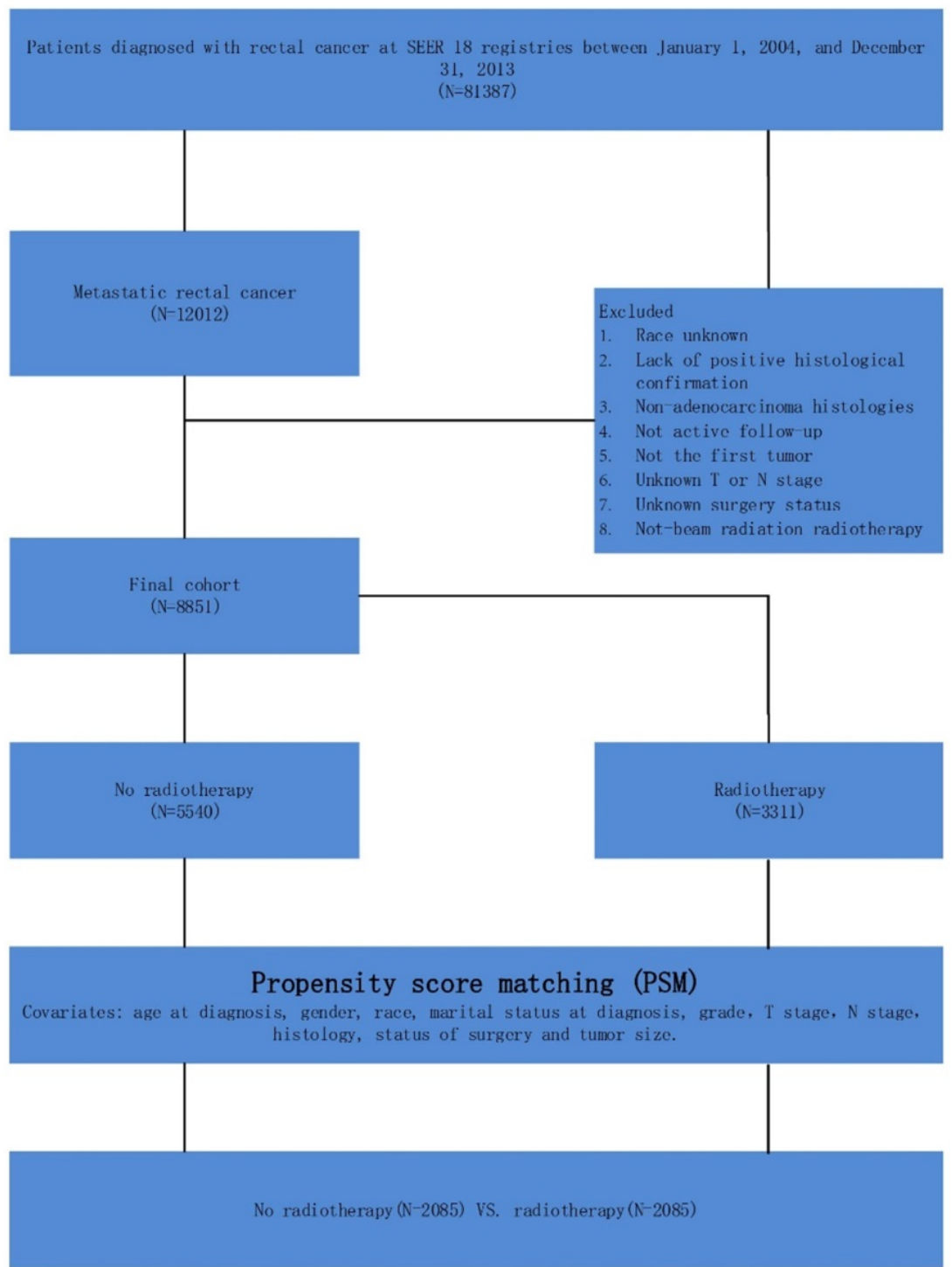

Figure 1. Schematic representation of patient population selected from SEER database.

increase in CSS (RT, HR=0.702, 95\% CI=0.665-0.741, $\mathrm{P}<0.001$, using no RT as a reference). In addition, we identified nine factors as independent protective factors included age, marital status at diagnosis, grade, AJCC $\mathrm{T}$ stage, AJCC $\mathrm{N}$ stage, histology, surgery status and tumor size. However, gender was not a prognostic factor according to univariate survival analysis $(\mathrm{P}=0.189)$. Propensity score matching was performed and resulted in 4170 patients. After PS matching, the 5-year CSSs of patients in the RT group and no-RT group were 22.0 and $13.5 \%$ respectively, a statistically significant difference in univariate analysis (Figure 2B, p<0.001).

\section{Patient characteristics and survival analyses of the surgery subgroup}

The overall cohort contained $5520 \mathrm{mRC}$ patients who had undergone surgery. Table 3 shows that patients less than 65 years old were more likely to receive radiotherapy $(59.3 \%$ vs. $72.4 \%, p<0.01)$. Both univariate analysis $(\mathrm{HR}=0.617,95 \% \mathrm{CI}=0.574-0.663$, $\mathrm{p}<0.001)$ and multivariate analysis $(\mathrm{HR}=0.654$, $95 \% \mathrm{CI}=0.607-0.704, \mathrm{p}<0.001)$ showed that RT was associated with significantly improved CSS (Table 4). Multivariate analysis also identified the following independent prognostic factors for CSS: patient age at diagnosis, patient marital status at diagnosis, tumor grade, tumor $\mathrm{T}$ stage, tumor $\mathrm{N}$ stage, tumor histology, patient surgery status and tumor size $(p<0.05)$. In addition, PS matching was performed using the above independent prognostic factors. The PS matching resulted in 2862 patients, and univariate analysis of the surgery subgroup showed that the 5-year survival rates of the RT and no-RT groups were significantly different (Figure 3A, 30.3 and 18.0\%, p<0.001). 
Table 1. Baseline characteristics of the study population.

\begin{tabular}{|c|c|c|c|c|c|c|}
\hline \multirow[t]{2}{*}{ Characteristic } & \multicolumn{3}{|l|}{ Before PS matching } & \multicolumn{3}{|l|}{ After PS matching } \\
\hline & No Radiotherapy (n (n\%)) & Radiotherapy (n (n\%)) & P value & No Radiotherapy (n (n\%)) & Radiotherapy (n (n\%)) & Pvalue \\
\hline Total & $5540(62.6)$ & $3311(37.4)$ & & $2085(50)$ & $2085(50)$ & \\
\hline Age (year) & & & $<0.001$ & & & 0.711 \\
\hline$<65$ & $3279(59.2)$ & $2233(67.4)$ & & 1449 (69.5) & $1460(70.0)$ & \\
\hline$\geq 65$ & $2261(40.8)$ & 1078 (32.6) & & $636(30.5)$ & $625(30.0)$ & \\
\hline Gender & & & 0.025 & & & 1.000 \\
\hline Male & $3288(59.4)$ & $2045(61.8)$ & & $1315(63.1)$ & $1315(63.1)$ & \\
\hline Female & $2252(40.6)$ & $1266(38.2)$ & & $770(36.9)$ & $770(36.9)$ & \\
\hline Race & & & 0.028 & & & 0.517 \\
\hline White & 4355 (78.6) & $2608(78.8)$ & & $1793(86.0)$ & 1805 (86.6) & \\
\hline Black & $606(10.9)$ & $360(10.9)$ & & $149(7.1)$ & $141(6.8)$ & \\
\hline Other & $560(10.1)$ & $342(10.3)$ & & $141(6.8)$ & $139(6.7)$ & \\
\hline Unknown & $19(0.3)$ & $1(0)$ & & $2(0.1)$ & 0 & \\
\hline Marital status at diagnosis & & & $<0.001$ & & & 0.837 \\
\hline Married & 2857 (51.6) & $1841(55.6)$ & & 1195 (57.3) & 1197 (57.4) & \\
\hline Unmarried & $2453(44.3)$ & 1373 (41.5) & & $854(41.0)$ & 847 (40.6) & \\
\hline Unknown & $230(4.2)$ & $97(2.9)$ & & $36(1.7)$ & $41(2.0)$ & \\
\hline Grade & & & 0.026 & & & 0.980 \\
\hline Grade I & $217(3.9)$ & $158(4.8)$ & & $54(2.6)$ & $58(2.8)$ & \\
\hline Grade II & 3395 (61.3) & $1990(60.1)$ & & $1414(67.8)$ & $1423(68.2)$ & \\
\hline Grade III & 1119 (20.2) & 635 (19.2) & & $372(17.8)$ & $370(17.7)$ & \\
\hline Grade IV & $110(2.0)$ & $54(1.6)$ & & $14(0.7)$ & $13(0.6)$ & \\
\hline Unknown & 699 (12.6) & $474((14.3)$ & & $231(11.1)$ & $221(10.6)$ & \\
\hline AJCC T-stage & & & $<0.001$ & & & 0.859 \\
\hline $\mathrm{T} 1$ & 844 (15.2) & $410(12.4)$ & & $260(12.5)$ & $263(12.6)$ & \\
\hline $\mathrm{T} 2$ & $199(3.6)$ & $170(5.1)$ & & $52(2.5)$ & $49(2.4)$ & \\
\hline $\mathrm{T} 3$ & 2984 (53.9) & 1941 (58.6) & & 1308 (62.7) & 1329 (63.7) & \\
\hline $\mathrm{T} 4$ & $1513(27.3)$ & $790(23.9)$ & & $465(22.3)$ & $444(21.3)$ & \\
\hline AJCC N-stage & & & $<0.001$ & & & 0.941 \\
\hline No & $1763(31.8)$ & 1113 (33.6) & & $655(31.4)$ & $646(31.0)$ & \\
\hline N1 & $1885(34.0)$ & 1365 (41.2) & & $880(42.2)$ & $881(42.3)$ & \\
\hline N2 & $1892(34.2)$ & $833(25.2)$ & & $550(26.4)$ & $558(26.8)$ & \\
\hline Histology & & & $<0.001$ & & & 0.772 \\
\hline Adenocarcinoma & $5210(94.0)$ & 3041 (91.8) & & 2016(96.7) & 2012 (96.5) & \\
\hline Mucinous adenocarcinoma & $243(4.4)$ & $211(6.4)$ & & $59(2.8)$ & $65(3.1)$ & \\
\hline Signet ring cell carcinoma & $87(1.6)$ & $59(1.8)$ & & $10(0.5)$ & $8(0.4)$ & \\
\hline Surgery & & & $<0.001$ & & & 0.974 \\
\hline No-surgery & $1976(35.7)$ & 1355 (40.9) & & $768(36.8)$ & $767(36.8)$ & \\
\hline Surgery & $3564(64.3)$ & $1956(59.1)$ & & 1317 (63.2) & $1318(63.2)$ & \\
\hline Tumor size $(\mathrm{cm})$ & & & $<0.001$ & & & 0.997 \\
\hline$\leq 5.0$ & $2260(40.8)$ & $1332(40.2)$ & & $927(44.5)$ & $925(44.4)$ & \\
\hline$>5.0$ & $1873(33.8)$ & $986(29.8)$ & & $612(29.4)$ & $612(29.4)$ & \\
\hline Unknown & $1407(25.4)$ & $993(30.0)$ & & $546(26.2)$ & $548(26.3)$ & \\
\hline
\end{tabular}

Table 2. Multivariate Cox regression analyses of CSS.

\begin{tabular}{|c|c|c|c|c|c|}
\hline \multirow[t]{2}{*}{ Covariate } & \multirow[t]{2}{*}{ Reference } & \multirow[t]{2}{*}{ Characteristic } & \multicolumn{3}{|c|}{ Cancer-specific survival } \\
\hline & & & HR $(95 \% \mathrm{CI})$ & SE & Pvalue \\
\hline Age (year) & $<65$ & $>=65$ & $1.602(1.519-1.688)$ & 0.027 & $<0.001$ \\
\hline \multirow[t]{3}{*}{ Race } & White & Black & $1.145(1.056-1.243)$ & 0.042 & 0.004 \\
\hline & & Other & $0.956(0.876-1.042)$ & 0.044 & 0.304 \\
\hline & & Unknown & $0.773(0.386-1.549)$ & 0.354 & 0.468 \\
\hline \multirow[t]{2}{*}{ Marital status at diagnosis } & Married & Unmarried & $1.224(1.161-1.291)$ & 0.027 & $<0.001$ \\
\hline & & Unknown & $0.876(0.751-1.022)$ & 0.079 & $<0.001$ \\
\hline \multirow[t]{4}{*}{ Grade } & Grade I & Grade II & $0.973(0.856-1.106)$ & 0.065 & 0.672 \\
\hline & & Grade III & $1.417(1.237-1.624)$ & 0.069 & $<0.001$ \\
\hline & & Grade IV & $1.718(1.377-2.145)$ & 0.113 & $<0.001$ \\
\hline & & Unknown & $1.094(0.949-1.262)$ & 0.073 & 0.215 \\
\hline \multirow[t]{3}{*}{ AJCC T-stage } & T1 & $\mathrm{T} 2$ & $0.804(0.691-0.936)$ & 0.077 & 0.005 \\
\hline & & T3 & $0.897(0.825-0.977)$ & 0.043 & 0.012 \\
\hline & & $\mathrm{T} 4$ & $1.17(1.071-1.279)$ & 0.045 & $<0.001$ \\
\hline \multirow[t]{2}{*}{ AJCC N-stage } & No & N1 & $1.044(0.978-1.114)$ & 0.033 & 0.197 \\
\hline & & N2 & $1.344(1.25-1.446)$ & 0.037 & $<0.001$ \\
\hline Radiotherapy & No radiotherapy & Radiotherapy & $0.702(0.665-0.741)$ & 0.028 & $<0.001$ \\
\hline \multirow[t]{2}{*}{ Histology } & Adenocarcinoma & Mucinous adenocarcinoma & $1.133(1.011-1.27)$ & 0.058 & 0.032 \\
\hline & & Signet ring cell carcinoma & $1.598(1.331-1.918)$ & 0.093 & $<0.001$ \\
\hline Surgery & No-surgery & Surgery & $0.450(0.421-0.481)$ & 0.034 & $<0.001$ \\
\hline \multirow[t]{2}{*}{ Tumor size $(\mathrm{cm})$} & $\leq 5.0$ & $>5.0$ & $1.031(0.970-1.097)$ & 0.031 & 0.326 \\
\hline & & Unknown & $1.120(1.046-1.200)$ & 0.035 & 0.001 \\
\hline
\end{tabular}


Table 3. Baseline characteristics of the surgery group.

\begin{tabular}{|c|c|c|c|c|c|c|}
\hline \multirow[t]{2}{*}{ Characteristic } & \multicolumn{3}{|l|}{ Before PS matching } & \multicolumn{3}{|l|}{ After PS matching } \\
\hline & $\begin{array}{l}\text { No Radiotherapy (n } \\
(\mathrm{n} \%))\end{array}$ & Radiotherapy (n (n\%)) & P value & No Radiotherapy (n (n $\%)$ ) & Radiotherapy (n (n\%)) & P value \\
\hline Total & $3564(64.6)$ & 1956(25.4) & & $1431(50)$ & $1431(50)$ & \\
\hline Age (year) & & & $<0.001$ & & & 0.771 \\
\hline$<65$ & $2112(59.3)$ & $1416(72.4)$ & & $1032(72.1)$ & 1025 (71.6) & \\
\hline$\geq 65$ & $1452(40.7)$ & $540(27.6)$ & & $399(27.9)$ & $406(28.4)$ & \\
\hline Gender & & & 0.105 & & & 0.758 \\
\hline Male & $2094(58.8)$ & $1193(61.0)$ & & $883(61.7)$ & $891(62.3)$ & \\
\hline Female & $1470(41.2)$ & $763(39.0)$ & & $548(38.3)$ & $540(37.7)$ & \\
\hline Race & & & 0.115 & & & 0.796 \\
\hline White & $2826(79.3)$ & $1578(80.7)$ & & $1207(84.3)$ & $1195(83.5)$ & \\
\hline Black & $349(9.8)$ & $183(9.4)$ & & $94(6.6)$ & $94(6.6)$ & \\
\hline Other & $376(10.5)$ & $194(9.9)$ & & $128(8.9)$ & $141(9.9)$ & \\
\hline Unknown & $13(0.4)$ & $1(0.1)$ & & $2(0.1)$ & $1(0.1)$ & \\
\hline Marital status at diagnosis & & & $<0.001$ & & & 0.743 \\
\hline Married & $1957(54.9)$ & $1184(60.5)$ & & $872(60.9)$ & $889(62.1)$ & \\
\hline Unmarried & 1465 (41.1) & $721(36.9)$ & & $527(36.8)$ & $514(35.9)$ & \\
\hline Unknown & $142(4.0)$ & $51(2.6)$ & & $32(2.2)$ & $28(2.0)$ & \\
\hline Grade & & & $<0.001$ & & & 0.114 \\
\hline Grade I & $127(3.6)$ & $87(4.4)$ & & $42(2.9)$ & $45(3.1)$ & \\
\hline Grade II & $2400(67.2)$ & $1269(64.9)$ & & $999(69.8)$ & $1006(70.3)$ & \\
\hline Grade III & $804(22.6)$ & $405(20.7)$ & & $316(22.1)$ & $292(20.4)$ & \\
\hline Grade IV & $95(2.7)$ & $37(1.9)$ & & $24(1.7)$ & $15(1.0)$ & \\
\hline Unknown & $138(3.9)$ & $158(8.1)$ & & $50(3.5)$ & $73(5.1)$ & \\
\hline AJCC T-stage & & & $<0.001$ & & & 0.979 \\
\hline $\mathrm{T} 1$ & $119(3.3)$ & $91(4.7)$ & & $51(3.6)$ & $48(3.4)$ & \\
\hline $\mathrm{T} 2$ & $136(3.8)$ & $107(5.5)$ & & $39(2.7)$ & $39(2.7)$ & \\
\hline T3 & $2337(65.6)$ & $1338(68.4)$ & & $1041(72.7)$ & $1050(73.4)$ & \\
\hline $\mathrm{T} 4$ & $972(27.3)$ & $420(21.5)$ & & $300(21.0)$ & $294(20.5)$ & \\
\hline AJCC N-stage & & & $<0.001$ & & & 0.917 \\
\hline No & $663(18.6)$ & $511(26.1)$ & & 299 (20.9) & $308(21.5)$ & \\
\hline N1 & 1155 (32.4) & $764(39.1)$ & & $575(40.2)$ & $572(40.0)$ & \\
\hline N2 & $1746(49.0)$ & $681(34.8)$ & & 557 (38.9) & $551(38.5)$ & \\
\hline Histology & & & $<0.001$ & & & 0.080 \\
\hline Adenocarcinoma & $3348(93.9)$ & $1758(89.9)$ & & $1340(93.6)$ & $1341(93.7)$ & \\
\hline Mucinous adenocarcinoma & $171(4.8)$ & $173(8.8)$ & & $75(5.2)$ & $84(5.9)$ & \\
\hline Signet ring cell carcinoma & $45(1.3)$ & $25(1.3)$ & & $16(1.1)$ & $6(0.4)$ & \\
\hline Tumor size $(\mathrm{cm})$ & & & $<0.001$ & & & 0.629 \\
\hline$\leq 5.0$ & $1807(50.7)$ & $967(49.4)$ & & $776(54.2)$ & $794(55.5)$ & \\
\hline$>5.0$ & $1422(39.9)$ & $571(29.2)$ & & $474(33.1)$ & $450(31.4)$ & \\
\hline Unknown & $335(9.4)$ & $418(21.4)$ & & $181(12.6)$ & $187(13.1)$ & \\
\hline
\end{tabular}

Table 4. Multivariate Cox regression analyses of surgery group.

\begin{tabular}{|c|c|c|c|c|c|}
\hline \multirow[t]{2}{*}{ Covariate } & \multirow[t]{2}{*}{ Reference } & \multirow[t]{2}{*}{ Characteristic } & \multicolumn{3}{|c|}{ Cancer-specific survival } \\
\hline & & & HR (95\%CI) & SE & P value \\
\hline Age (year) & $<65$ & $>=65$ & $1.580(1.474-1.693)$ & 0.058 & $<0.001$ \\
\hline \multirow[t]{3}{*}{ Race } & White & Black & $1.059(0.946-1.185)$ & 0.057 & 0.320 \\
\hline & & Other & $0.917(0.820-1.025)$ & 0.410 & 0.128 \\
\hline & & Unknown & $0.795(0.356-1.773)$ & 0.035 & 0.575 \\
\hline \multirow[t]{2}{*}{ Marital status at diagnosis } & Married & Unmarried & $1.223(1.142-1.310)$ & 0.103 & $<0.001$ \\
\hline & & Unknown & $0.970(0.792-1.188)$ & 0.087 & 0.769 \\
\hline \multirow[t]{4}{*}{ Grade } & Grade I & Grade II & $0.833(0.702-0.988)$ & 0.091 & 0.035 \\
\hline & & Grade III & $1.215(1.016-1.454)$ & 0.135 & 0.033 \\
\hline & & Grade IV & $1.437(1.103-1.872)$ & 0.116 & 0.007 \\
\hline & & Unknown & $0.854(0.680-1.071)$ & 0.126 & 0.172 \\
\hline \multirow[t]{3}{*}{ AJCC T-stage } & T1 & $\mathrm{T} 2$ & $0.773(0.604-0.991)$ & 0.097 & 0.042 \\
\hline & & T3 & $0.921(0.761-1.114)$ & 0.101 & 0.395 \\
\hline & & T4 & $1.203(0.987-1.467)$ & 0.05 & 0.067 \\
\hline \multirow[t]{2}{*}{ AJCC N-stage } & No & N1 & $1.126(1.020-1.242)$ & 0.048 & 0.018 \\
\hline & & N2 & $1.477(1.343-1.624)$ & 0.038 & $<0.001$ \\
\hline Radiotherapy & No radiotherapy & Radiotherapy & $0.654(0.607-0.705)$ & 0.069 & $<0.001$ \\
\hline \multirow[t]{2}{*}{ Histology } & Adenocarcinoma & Mucinous adenocarcinoma & $1.105(0.966-1.264)$ & 0.136 & 0.145 \\
\hline & & Signet ring cell carcinoma & $1.963(1.503-2.564)$ & 0.037 & $<0.001$ \\
\hline \multirow[t]{2}{*}{ Tumor size $(\mathrm{cm})$} & $\leq 5.0$ & $>5.0$ & $1.021(0.949-1.098)$ & 0.053 & 0.576 \\
\hline & & Unknown & $1.216(1.096-1.350)$ & 0.058 & $<0.001$ \\
\hline
\end{tabular}




\section{A}
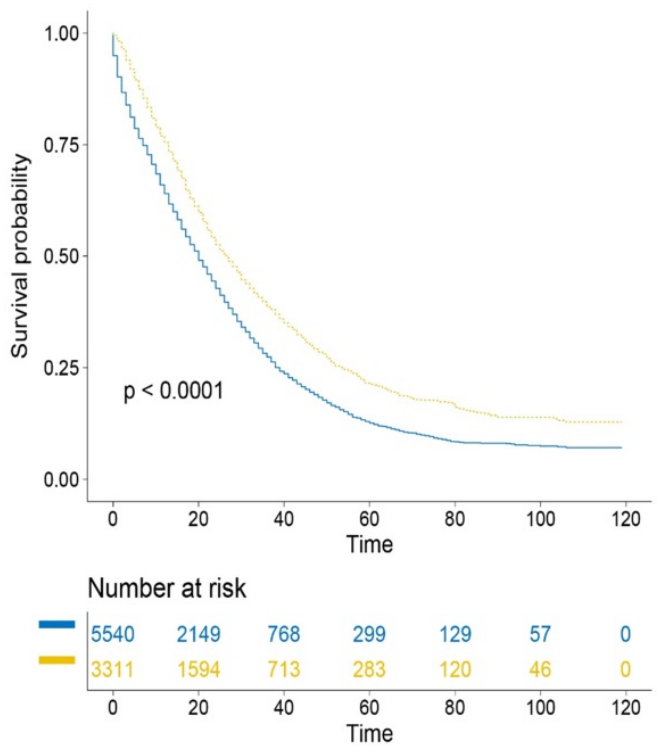

B
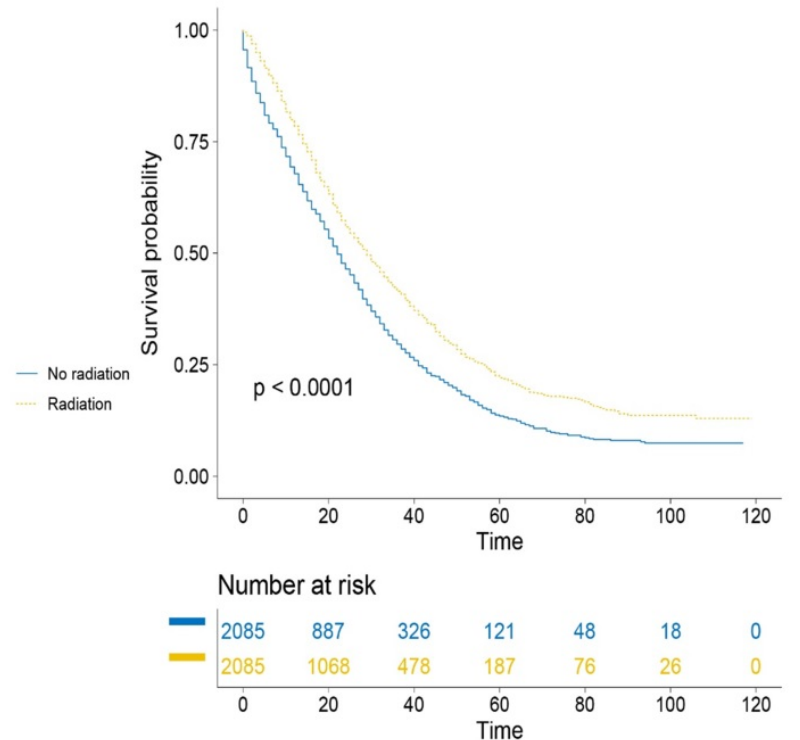

Figure 2. Survival curves of $m R C$ patients in SEER database (A). Before PS matching: the 5-year CSSs of patients in RT group and no-RT group were 21.2 and $12.6 \%$, respectively, of which the difference was statistically significant $(P<0.001)$, (B). After PS matching: the 5-year CSSs of patients in RT group and no-RT group were 22.0 and $13.5 \%$, respectively, of which the difference was statistically significant $(P<0.001)$.

A

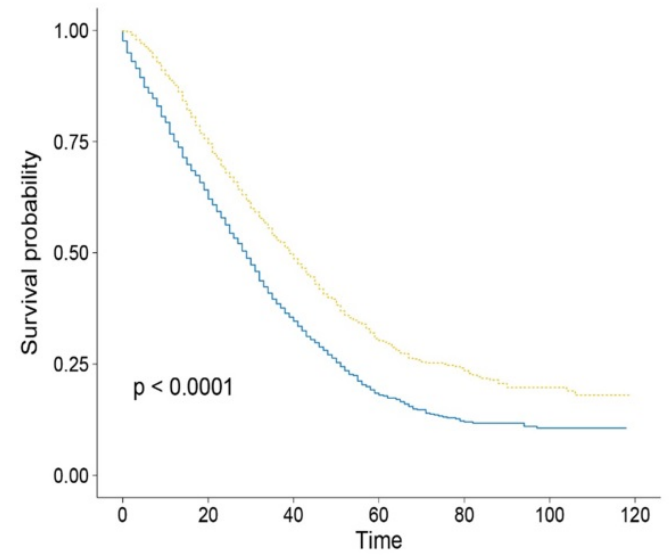

Number at risk

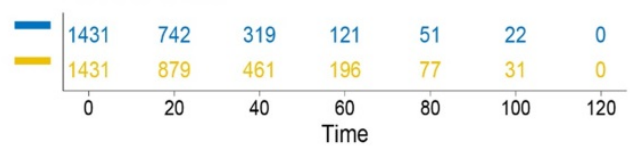

$\mathrm{B}$
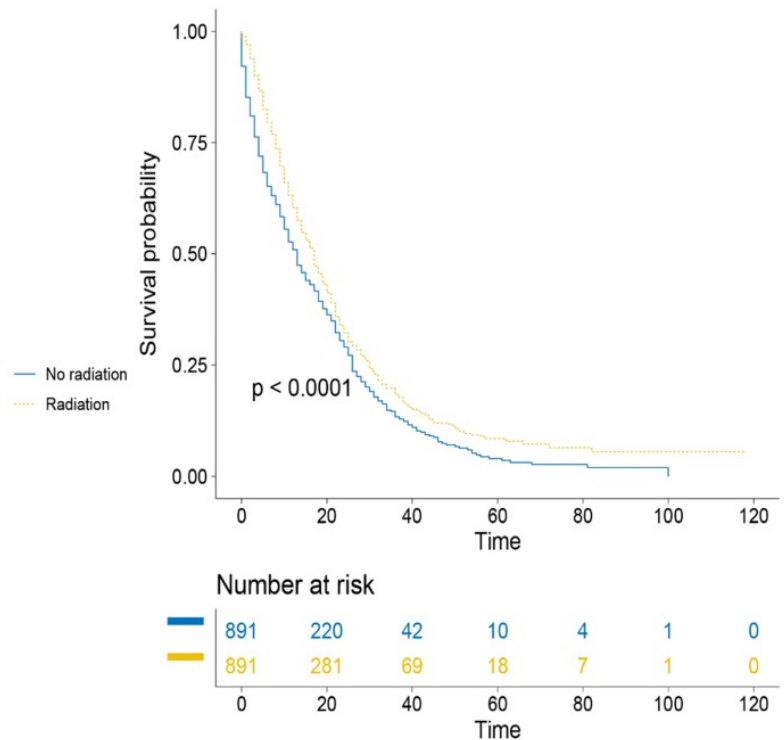

Figure 3. Survival curves of $m R C$ patients in SEER database after PS matching (A). In surgery group: the 5-year CSSs of patients in RT group and no-RT group were 30.3 and $18.0 \%$, respectively, of which the difference was statistically significant $(P<0.001)$, (B). In no-surgery group: the 5 -year CSSs of patients in RT group and no-RT group were 8.4 and $4.0 \%$, respectively, of which the difference was statistically significant $(P<0.001)$.

\section{Patient characteristics and survival analyses of no-surgery subgroup}

The overall cohort contained $3331 \mathrm{mRC}$ patients who had not received surgery. Table 5 shows that patients with $\mathrm{T} 3$ stage tumors were more likely to receive radiotherapy $(32.7 \%$ vs. $44.5 \%, \mathrm{p}<0.01)$. Both univariate analysis $(\mathrm{HR}=0.785,95 \% \mathrm{CI}=0.723-0.852$, $\mathrm{p}<0.001)$ and multivariate analysis $(\mathrm{HR}=0.779$, $95 \% \mathrm{CI}=0.717-0.847, \mathrm{p}<0.001)$ show that $\mathrm{RT}$ was associated with significantly improved CSS (Table 6).
Multivariate analysis also identified the following independent prognostic factors: patient age at diagnosis, patient race, patient marital status at diagnosis, tumor grade, tumor $\mathrm{T}$ stage, tumor histology and status of surgery $(\mathrm{p}<0.05)$. In addition, PS matching was performed using the above independent prognostic factors. The PS matching resulted in 1782 patients and univariate analysis of the no surgery subgroup showed that the 5-year survival rates of the RT and no-RT groups were also significantly different (Figure 3B, 8.4 vs. $4.0 \%$, p<0.01). 
Table 5. Baseline characteristics of the no-surgery group.

\begin{tabular}{|c|c|c|c|c|c|c|}
\hline \multirow[t]{2}{*}{ Characteristic } & \multicolumn{3}{|l|}{ Before PS matching } & \multicolumn{3}{|l|}{ After PS matching } \\
\hline & No Radiotherapy (n (n\%)) & Radiotherapy (n (n\%)) & P value & No Radiotherapy (n (n\%)) & Radiotherapy (n (n\%)) & P value \\
\hline Total & $1976(59.3)$ & $1355(40.7)$ & & $891(50)$ & $891(50)$ & \\
\hline Age (year) & & & 0.475 & & & 0.623 \\
\hline$<65$ & $1167(59.1)$ & $817(60.3)$ & & $561(63.0)$ & $571(64.1)$ & \\
\hline$\geq 65$ & $809(40.9)$ & $538(39.7)$ & & $330(37.0)$ & $320(35.9)$ & \\
\hline Gender & & & 0.153 & & & 0.922 \\
\hline Male & $1194(60.4)$ & $852(62.9)$ & & $557(62.5)$ & $555(62.3)$ & \\
\hline Female & $782(39.6)$ & $503(37.1)$ & & $334(37.5)$ & $336(37.7)$ & \\
\hline Race & & & 0.088 & & & 0.852 \\
\hline White & $1529(77.4)$ & $1030(76.0)$ & & $731(82.0)$ & $722(81.0)$ & \\
\hline Black & $257(13.0)$ & $177(13.1)$ & & $84(9.4)$ & $90(10.1)$ & \\
\hline Other & $184(9.3)$ & $148(10.9)$ & & $76(8.5)$ & $79(8.9)$ & \\
\hline Unknown & $6(0.3)$ & 0 & & 0 & 0 & \\
\hline Marital status at diagnosis & & & 0.116 & & & 0.999 \\
\hline Married & $900(45.5)$ & $657(48.5)$ & & $422(47.4)$ & $423(47.5)$ & \\
\hline Unmarried & $988(50.0)$ & $652(48.1)$ & & $452(40.7)$ & $451(50.6)$ & \\
\hline Unknown & $88(4.5)$ & $46(3.4)$ & & $17(1.9)$ & $17(1.9)$ & \\
\hline Grade & & & 0.014 & & & 0.650 \\
\hline Grade I & $90(4.6)$ & $71(5.2)$ & & $38(4.3)$ & $34(3.8)$ & \\
\hline Grade II & $995(50.4)$ & $721(53.2)$ & & $499(56.0)$ & $485(54.4)$ & \\
\hline Grade III & 315 (15.9) & $230(17.0)$ & & 135 (15.2) & $135(15.2)$ & \\
\hline Grade IV & $15(0.8)$ & $17(1.3)$ & & $5(0.6)$ & $10(1.1)$ & \\
\hline Unknown & $561(28.4)$ & $316(23.3)$ & & $214(24.0)$ & $227(25.5)$ & \\
\hline AJCC T-stage & & & $<0.001$ & & & 0.198 \\
\hline $\mathrm{T} 1$ & $725(36.7)$ & $319(23.5)$ & & $271(30.4)$ & $252(28.3)$ & \\
\hline $\mathrm{T} 2$ & $63(3.2)$ & $63(4.6)$ & & $18(2.0)$ & $32(3.6)$ & \\
\hline T3 & $647(32.7)$ & $603(44.5)$ & & $373(41.9)$ & $379(42.5)$ & \\
\hline $\mathrm{T} 4$ & $541(27.4)$ & $370(27.3)$ & & $229(25.7)$ & $228(25.6)$ & \\
\hline AJCC N-stage & & & $<0.001$ & & & 0.395 \\
\hline No & $1100(55.7)$ & $602(44.4)$ & & $446(50.1)$ & $442(49.6)$ & \\
\hline N1 & $730(36.9)$ & $601(44.4)$ & & $381(42.8)$ & $398(44.7)$ & \\
\hline N2 & $146(7.4)$ & $152(11.2)$ & & $64(7.2)$ & $51(5.7)$ & \\
\hline Histology & & & 0.324 & & & 0.075 \\
\hline Adenocarcinoma & $1862(94.2)$ & $1283(94.7)$ & & $861(96.6)$ & $858(96.3)$ & \\
\hline Mucinous adenocarcinoma & $72(3.6)$ & $38(2.8)$ & & $22(2.5)$ & $15(1.7)$ & \\
\hline Signet ring cell carcinoma & $42(2.1)$ & $34(2.5)$ & & $8(0.9)$ & $18(2.0)$ & \\
\hline Tumor size $(\mathrm{cm})$ & & & $<0.001$ & & & 0.695 \\
\hline$\leq 5.0$ & $453(22.9)$ & 365 (26.9) & & $220(24.7)$ & $220(24.7)$ & \\
\hline$>5.0$ & $451(22.8)$ & $415(30.6)$ & & $235(26.4)$ & $250(28.1)$ & \\
\hline Unknown & $1072(54.3)$ & $575(42.4)$ & & $436(48.9)$ & $421(47.3)$ & \\
\hline
\end{tabular}

Table 6. Multivariate Cox regression analyses of no-surgery group.

\begin{tabular}{|c|c|c|c|c|c|}
\hline \multirow[t]{2}{*}{ Covariate } & \multirow[t]{2}{*}{ Reference } & \multirow[t]{2}{*}{ Characteristic } & \multicolumn{3}{|c|}{ Cancer-specific survival } \\
\hline & & & HR (95\%CI) & SE & P value \\
\hline Age (year) & $<65$ & $>=65$ & $1.618(1.490-1.758)$ & 0.042 & $<0.001$ \\
\hline \multirow[t]{3}{*}{ Race } & White & Black & $1.233(1.095-1.389)$ & 0.061 & 0.001 \\
\hline & & Other & $1.019(0.888-1.170)$ & 0.070 & 0.786 \\
\hline & & Unknown & $0.692(0.172-2.780)$ & 0.710 & 0.604 \\
\hline \multirow[t]{2}{*}{ Marital status at diagnosis } & Married & Unmarried & $1.219(1.121-1.325)$ & 0.042 & $<0.001$ \\
\hline & & Unknown & $0.787(0.620-0.998)$ & 0.121 & 0.048 \\
\hline \multirow[t]{4}{*}{ Grade } & Grade I & Grade II & 1.152(0.948-1.399) & 0.099 & 0.155 \\
\hline & & Grade III & $1.688(1.366-2.086)$ & 0.108 & $<0.001$ \\
\hline & & Grade IV & $2.262(1.449-3.530)$ & 0.227 & $<0.001$ \\
\hline & & Unknown & $1.320(1.078-1.617)$ & 0.103 & 0.007 \\
\hline \multirow[t]{3}{*}{ AJCC T-stage } & T1 & $\mathrm{T} 2$ & $0.883(0.703-1.109)$ & 0.116 & 0.284 \\
\hline & & T3 & $0.889(0.803-0.985)$ & 0.052 & 0.025 \\
\hline & & $\mathrm{T} 4$ & $1.170(1.051-1.302)$ & 0.055 & 0.004 \\
\hline \multirow[t]{2}{*}{ AJCC N-stage } & No & N1 & $0.980(0.896-1.071)$ & 0.046 & 0.651 \\
\hline & & N2 & $1.060(0.912-1.231)$ & 0.077 & 0.451 \\
\hline Radiotherapy & No radiotherapy & Radiotherapy & $0.779(0.717-0.847)$ & 0.042 & $<0.001$ \\
\hline \multirow[t]{2}{*}{ Histology } & Adenocarcinoma & Mucinous adenocarcinoma & $1.297(1.042-1.614)$ & 0.112 & 0.020 \\
\hline & & Signet ring cell carcinoma & 1.397(1.083-1.801) & 0.130 & 0.010 \\
\hline \multirow[t]{2}{*}{ Tumor size (cm) } & $\leq 5.0$ & $>5.0$ & $1.054(0.939-1.184)$ & 0.059 & 0.371 \\
\hline & & Unknown & $1.088(0.984-1.203)$ & 0.051 & 0.101 \\
\hline
\end{tabular}

\section{Discussion}

$\mathrm{RT}$ is reported to be used as palliative treatment to effectively relieve pelvic symptoms such as bleeding, pain and other pelvic symptoms in the clear majority of patients with symptomatic rectal cancer [16-19]. According to the NCCN clinical practice guidelines on rectal cancer (version 3.2017), in the case 
of $\mathrm{mRC}$, RT was only recommended for patients to reduce the pelvic recurrence. And Yamada S et al.[20] suggested that Carbon-ion radiation therapy may be an effective treatment for locally recurrent rectal cancer. The survival benefit of RT, however, was inconclusive, and the results were restricted by the amount of the samples [21-24]. In addition, prospective studies demonstrating a survival benefit for patients with $\mathrm{mRC}$ receiving palliative RT do not exist. SEER is a comprehensive population-based database in the United States that includes disease stage at initial diagnosis, initial treatments performed, and accurate data regarding patient survival. In this study, a total of 8851 patients with $\mathrm{mRC}$ were identified with initial presentation between 2004 and 2013 in the SEER database and our analyses indicated that RT was associated with an improved survival benefit.

An interesting phenomenon in the overall cohort was that patient age was negatively associated with the receipt of $\mathrm{RT}$, and one explanation for this phenomenon was the presence of comorbidities. RT is usually used for symptoms management and few reports suggest decreased efficacy or increased toxicity when palliative RT is used in older patients. However, the reasons behind this phenomenon are unclear and deserve further investigation even in the oldest patients [25-27].

For now, surgery provides the best treatment for rectal cancer, and chemotherapy can be a valuable adjunct therapy when given in a (neo-)adjuvant fashion or as conversion therapy to reduce tumor size in the initially unresectable tumors of $\mathrm{mRC}$ patients. RT is a successful, time-efficient, well-tolerated, and cost-effective intervention that is an indispensable component of palliative oncology care, and the clear benefits of RT for the treatment of stage II/III rectal cancer have be identified. However, the role of RT in the treatment of $\mathrm{mRC}$ is not yet clear.

There is very little data concerning the survival benefit of RT in $\mathrm{mRC}$, and the earliest exploration we can found is in 2010, when Kim and his colleagues [28] suggested that RT could significantly reduce the pelvic failure rate but its influence on overall survival was unclear with a retrospective clinical study containing only 89 patients diagnosed with synchronous liver metastasis. In 2012, Chang CY et al. [24] compared the effect of concurrent postoperative RT with chemotherapy to postoperative systemic chemotherapy without RT by the analyses of 69 patients with $\mathrm{mRC}$ after complete resection and found both no apparent survival benefit and reduced local recurrence of postoperative $\mathrm{RT}$ in $\mathrm{mRC}$ after complete removal of tumors. Also in 2012, a study in Korea believed that the survival benefit of RT in mRC was not apparent after an analysis of 68 patients with metastatic rectal cancer [29]. Similarly, Min et al. [22] analyzed 176 patients with mRC who underwent TME and reported that pelvic RT might have benefits in locoregional control, yet the survival difference was not significantly different between the RT and no-RT groups after surgery. From the above it can be seen that the previous studies suggested that the survival benefit in mRC was not apparent or inconclusive. However, we did not agree with the conclusions and we think their results were restricted by the amount of samples. In 2001, Crane et al. [30] found that chemoradiation combined with surgery had an improved 2-year survival compared with radiation alone, which is in good agreement with our analysis. Before PS matching, the 5-year CSSs in the RT group and the no-RT group for the overall cohort were $21.2 \%$ and $12.6 \%$, respectively $(\mathrm{p}<0.01)$, similar to outcomes after PS matching. The 5-year CSS for patients who had received RT was $30.3 \%$ compared with $18.0 \%$ for those patients not treated with RT in the surgery subgroup ( $\mathrm{p}<0.001)$ and the improved 5-year CSS was higher than that of the overall cohort. In addition, the no-surgery subgroup also showed an improved CSS, but not as obvious as the surgery subgroup, which is consistent with the study above: mRC patients who have received surgery are more likely to be treated with RT than those who have not received surgery. In clinical practice, many clinicians recommend postoperative RT in $\mathrm{mRC}$ patients after curative resection based on the NCCN guidelines. We strongly agree with this way according the result of our analyses. RT can not only relieve pelvic symptoms and reduce local recurrence but also provide apparent survival benefit after surgery. Beyond that, the adverse effects offered by radiotherapy are often favorable when compared to those caused by chemotherapy. Except for the increased fatigue, the toxicity of radiotherapy is limited to the normal tissues [31]. And most common radiotherapy adverse effects resolve within 4 weeks after the completion of treatment, previous studies also showed that quality of life could return to baseline within 1 month $[32,33]$. A 12-year follow-up of a multicenter randomized controlled trial [34] showed that the improved CSS with radiotherapy was weakened by an increase in other causes of death, we therefore believe more research is needed to determine a more accurate role for RT.

What might account for the survival benefit of palliative RT to $\mathrm{mRC}$ ? Patients with metastatic breast cancer, renal cell carcinoma, prostate cancer, lung cancer, and primary brain tumors are reported to have a survival benefit with the use of RT to treat the primary or metastatic disease by decreasing tumor 
burden [35-39]. RT could eliminate the primary tumor burden of rectal cancer, which reduced the probability of death by uncontrolled local disease progression, and a reduced tumor burden might reduce the source of cancer cells that could metastasize by "self-seeding" [40]. Therefore, RT may reduce the number of circulating rectal cancer cells. Many studies have documented the myriad mechanisms through which RT can increase the sensitivity of tumor cells to direct T-cell-mediated killing [41]. Therefore, we also think RT-enhanced immune function may be responsible for the improvement of survival. And Low dose radiation has been shown to induce biological responses like inflammatory responses, innate immune system activation, the repair of cellular DNA and protein damage which are the adaptive response [42]. Still, the mechanism underlying the survival benefit of $\mathrm{RT}$ to $\mathrm{mRC}$ remains unknown. In addition, RT is also reported to exert local and distant effects on tumor cells migration through mechanisms including vascular damage, EMT induction, cytokine production and macrophage-induced metastasis [43-45], leading us to believe that the mechanisms underlying the impact of RT to metastatic disease are complicated and include multiple factors. Above all, we strongly believe that the mechanisms and interactions behind this phenomenon deserve further and careful investigation.

Our study had several limitations when using the SEER database for analyses. The first limitation is that the SEER database does not collect detailed follow-up information, so we were unable to identify whether the patients originally presented with $\mathrm{mRC}$ or developed metastatic disease after the diagnosis of rectal cancer, and then received palliative RT. There is a possibility that the efficacy of palliative RT differs between the patients who originally presented with $\mathrm{mRC}$ and those who developed metastatic disease after the diagnosis of rectal cancer. Next, SEER reporting does not specify the regions where RT was administered (i.e., the pelvis, the primary tumor, metastatic sites or a combination). The regions to which RT was administered might also be associated with the efficacy of RT. Finally, the RT modalities used were heterogeneous. Although there is no evidence of a survival difference between different irradiation modalities in the literature, the heterogeneity in different treatment modalities may affect the outcomes. Despite these drawbacks, we believe our analyses provide a better understanding of the role of palliative RT in mRC patients and could induce more relevant studies that will direct the clinical application of RT in rectal cancer patients with metastasis in the future.

\section{Conclusion}

Currently, surgery and chemotherapy are the primary treatments for $\mathrm{mRC}$. However, our analyses using the SEER database suggest that RT could provide significant survival benefits for patients with stage IV rectal cancer, and we thus argue that standard local treatment should not be disregarded in patients with metastatic disease. We also believe these results need to be tested in more clinical trials to elucidate the biological mechanisms by which RT affects survival, to identify which patients might best benefit from RT, and to accurately define the role of $\mathrm{RT}$ in the comprehensive treatment of $\mathrm{mRC}$ patients.

\section{Acknowledgments}

We have great respect for the work that goes into compiling and maintaining the Surveillance, Epidemiology, and End Results Program (SEER) tumor registries, including the interpretation and reporting of these data and so on.

\section{Funding}

The National Natural Science Foundation of China (Grant Nos. 81702353 and 81772599) and Shanghai Municipal Natural Science Foundation (17ZR1406400) supported this work. The funders had no role in the study design, data collection and analysis, decision to publish, or preparation of the manuscript.

\section{Data availability statement}

The data of this study can be freely available from the Surveillance, Epidemiology, and End Results Program database.

\section{Competing Interests}

The authors have declared that no competing interest exists.

\section{References}

1. Siegel RL, Miller KD, Fedewa SA, Ahnen DJ, Meester RGS, Barzi A, et al. Colorectal cancer statistics, 2017. Ca A Cancer Journal for Clinicians 2017; 67: 104-17.

2. Byeon JS, Yang SK, Kim TI, Kim WH, Lau JY, Leung WK, et al. Colorectal neoplasm in asymptomatic Asians: a prospective multinational multicenter colonoscopy survey. Gastrointestinal Endoscopy 2007; 65: 1015-22.

3. Sung JJY, Ng SC, Chan FKL, Chiu HM, Kim HS, Matsuda T, et al. An updated Asia Pacific Consensus Recommendations on colorectal cancer screening. Gut 2015; 64: 121.

4. Labianca R, Beretta G, Kildani B, Milesi L, Merlin F, Mosconi S, et al. Colon cancer. Critical Reviews in Oncology/Hematology 2010; 74: 106-33.

5. Listed N. StatBite. At what stage are colorectal cancer patients diagnosed? Journal of the National Cancer Institute 2009; 101: 1114-.

6. Tsoulfas G, Pramateftakis MG. Management of rectal cancer and liver metastatic disease: which comes first? International Journal of Surgical Oncology 2012,(2012-05-14) 2012; 2012: 196908

7. Eadens MJ, Grothey A. Curable metastatic colorectal cancer. Current Oncology Reports 2011; 13: 168.

8. Guren MG, Kørner H, Pfeffer F, Myklebust TÅ, Eriksen MT, Edna TH, et al. Nationwide improvement of rectal cancer treatment outcomes in Norway, 1993-2010. Acta Oncologica 2015; 54: 1-9. 
9. Fakih MG. Metastatic colorectal cancer: current state and future directions. Journal of Clinical Oncology Official Journal of the American Society of Clinical Oncology 2015; 33: 1809-24.

10. Sun J, Pichler P, Dowling J, Menk F, Stanwell P, Arm J, et al. Estimation of an optimal utilisation rate for palliative radiotherapy in newly diagnosed cancer patients. Clinical Oncology 2010; 22: 56-64.

11. Ly BH, Vlastos G, Rapiti E, Vinhhung V, Nouyen NP. Local-regional radiotherapy and surgery is associated with a significant survival advantage in metastatic breast cancer patients. Tumori 2010; 96: 947-54.

12. $\mathrm{Hu} \mathrm{J}$, Lin K, Jing G, Hu W, Guan X, Lu JJ. Use of Radiation Therapy in Metastatic Nasopharyngeal Cancer Improves Survival: A SEER Analysis. Sci Rep 2017; 7: 721

13. Shao YH, Kim S, Moore DF, Shih W, Lin Y, Stein M, et al. Cancer-specific survival after metastasis following primary radical prostatectomy compared with radiation therapy in prostate cancer patients: results of a population-based, propensity score-matched analysis. European Urology 2014; 65: 693-700.

14. Glynn RJ, Schneeweiss S, Stürmer T. Indications for propensity scores and review of their use in pharmacoepidemiology. Basic \& Clinical Pharmacology \& Toxicology 2006; 98: 253 .

15. Brookhart MA, Schneeweiss S, Rothman KJ, Glynn RJ, Avorn J, Stürmer T, Variable Selection for Propensity Score Models. American Journal of Epidemiology 2006; 163: 1149

16. Cameron MG, Kersten C, Vistad I, Van HR, Weyde K, Undseth C, et al. Palliative pelvic radiotherapy for symptomatic rectal cancer - a prospective multicenter study. Radiotherapy \& Oncology 2015; 115: 314-20.

17. Chia D, Lu J, Leong C, Wong L, Tan G, Tey J. Efficacy of Palliative Radiation Therapy in Symptomatic Rectal Cancer in the Modern Era. Radiotherapy \& Oncology 2016; 84: S342-S.

18. Sun HB, Park W, Choi DH, Nam H, Kang WK, Park YS, et al. Palliative radiotherapy in patients with a symptomatic pelvic mass of metastatic colorectal cancer. Radiation Oncology,6,1(2011-05-21) 2011; 6: 1-8.

19. Picardi V, Deodato F, Guido A, Giaccherini L, Macchia G, Frazzoni L, et al. Palliative Short-Course Radiation Therapy in Rectal Cancer: A Phase 2 Study. International Journal of Radiation Oncology*Biology*Physics 2016; 95: 1184-90

20. Yamada S, Kamada T, Ebner DK, Shinoto M, Terashima K, Isozaki Y, et al. Carbon-Ion Radiation Therapy for Pelvic Recurrence of Rectal Cancer. International Journal of Radiation Oncology Biology Physics 2016; 96: 93-101.

21. An HJ, Yu CS, Yun SC, Kang BW, Hong YS, Lee JL, et al. Adjuvant chemotherapy with or without pelvic radiotherapy after simultaneous surgical resection of rectal cancer with liver metastases: analysis of prognosis and patterns of recurrence. International Journal of Radiation Oncology Biology Physics 2012; 84: 73.

22. Kim MJ, Kim SJ, Park SC, Kim DY, Park JW, Ryoo SB, et al Adjuvant radiotherapy for the treatment of stage IV rectal cancer after curative resection:A propensity score-matched analysis and meta-analysis. Medicine 2016; 95: e4925.

23. Lee JH, Jo IY, Lee JH, Yoon SC, Kim YS, Choi BO, et al. The role of postoperative pelvic radiation in stage IV rectal cancer after resection of primary tumor. Radiation Oncology Journal 2012; 30: 205-12.

24. Chang $\mathrm{CY}$, Kim HC, Park YS, Park JO, Choi DH, Park HC, et al. The effect of postoperative pelvic irradiation after complete resection of metastatic rectal cancer. Journal of Surgical Oncology 2012; 105: 244

25. Mitsuhashi N, Hayakawa K, Yamakawa M, Sakurai H, Saito Y, Hasegawa M, et al. Cancer in patients aged 90 years or older: radiation therapy. Radiology 1999; 211: 829-33.

26. Wu J, McCloskey S, Steinberg ML. Chapter 7 - Radiation Therapy for the Older Patient. In: Management of Cancer in the Older Patient. Philadelphia: W.B. Saunders; 2012: pp. 65-77.

27. Zachariah B, Balducci L, Venkattaramanabalaji GV, Casey L, Greenberg HM, Delregato JA. Radiotherapy for cancer patients aged 80 and older: a study of effectiveness and side effects. International Journal of Radiation Oncology Biology Physics 1997; 39: 1125-9.

28. Kim JW, Yong BK, Kim NK, Min BS, Sang JS, Ahn JB, et al. The role of adjuvant pelvic radiotherapy in rectal cancer with synchronous liver metastasis: a retrospective study. Radiation oncology (London, England) 2010; 5: 75 .

29. Chang CY, Kim HC, Park YS, Park JO, Choi DH, Park HC, et al. The effect of postoperative pelvic irradiation after complete resection of metastatic rectal cancer. Journal of Surgical Oncology 2012; 105: 244-8.

30. Crane CH, Janjan NA, Abbruzzese JL, Curley S, Vauthey J, Sawaf HB, et al. Effective pelvic symptom control using initial chemoradiation without colostomy in metastatic rectal cancer. International Journal of Radiation Oncology Biology Physics 2001; 49: 107.

31. Lutz S, Korytko T, Nguyen J, Khan L, Chow E, Corn B. Palliative radiotherapy: when is it worth it and when is it not? Cancer Journal 2010; 16: 473

32. Brown P, Clark MM, Atherton P, Huschka M, Sloan JA, Gamble G, et al. Will improvement in quality of life (QOL) impact fatigue in patients receiving radiation therapy for advanced cancer? American Journal of Clinical Oncology 2006; 29: 52-8

33. Li LM, Kathy Meers RN, Anita Capurso RN, Engebretson TO, Glicksman AS. The Impact of Radiation Therapy on Quality of Life in Patients withCancer. Cancer Practice 1998; 6: 237-42.

34. W vG, CA M, ID N, EM K, H P, T W, et al. Preoperative radiotherapy combined with total mesorectal excision for resectable rectal cancer: 12-year follow-up of the multicentre, randomised controlled TME trial. Lancet Oncology 2011; 12: 575-82.

35. Scodan RL, Stevens D, Tubiana-Hulin M, Lande BDL, Cohen-Solal C, Brain E, et al. Breast cancer with synchronous metastases: Impact of locoregional radiotherapy (LRR) on survival. Journal of Clinical Oncology 2008; 26: 1375-81.

36. Cho Y, Chang JS, Rha KH, Hong SJ, Choi YD, Ham WS, et al. Does Radiotherapy for the Primary Tumor Benefit Prostate Cancer Patients with Distant Metastasis at Initial Diagnosis? Plos One 2016; 11: e0147191.

37. Majewski W, Tabor M, Banaszek P, Glowacki G, Rokicki W, Tukiendorf A. The efficacy of stereotactic radiotherapy for metastases from renal cell carcinoma. Neoplasma 2016; 63: 99-106.

38. Nitta T, Sato K. Prognostic implications of the extent of surgical resection in patients with intracranial malignant gliomas. Cancer 1995; 75: 2727-31.

39. Zhu H, Zhou Z, Wang Y, Bi N, Feng $\mathrm{Q}$, Li J, et al. Thoracic radiation therapy improves the overall survival of patients with extensive-stage small cell lung cancer with distant metastasis. Cancer 2011; 117: 5423-31.

40. Comen E, Norton L, Massagué J. Clinical implications of cancer self-seeding. Nature Reviews Clinical Oncology 2011; 8: 369-77.

41. Burnette B, Weichselbaum RR. The Immunology of Ablative Radiation. Seminars in Radiation Oncology 2015; 25: 40-5.

42. Nezih H, Zafer C, Zacharenia N, Aysegul C, Eyup Ilker S. Radiation triggering immune response and inflammation. Cancer Letters 2015; 368: 156.

43. Timaner M, Bril R, Kaidar-Person O, Chen RT, Alishekevitz D, Kotsofruk R, et al. Dequalinium blocks macrophage-induced metastasis following local radiation. Oncotarget 2015; 6: 27537-54.

44. Vilalta M, Rafat M, Graves EE. Effects of radiation on metastasis and tumor cell migration. Cellular \& Molecular Life Sciences Cmls 2016; 73: 2999.

45. Kuk PJ, Jin JS, Kang SW, Sunhoo P, Sang-Gu H, Wun-Jae K, et al. Establishment of animal model for the analysis of cancer cell metastasis during radiotherapy. Radiation Oncology 2012; 7: 153 